\title{
¿Psicología positiva para afrontar la radicalización y el terrorismo? Un análisis del discurso de Obama en el Cairo
}

\section{A positive psychology to cope with radicalisation and terrorism? A case study of the speech by Barack Obama at Cairo}

\author{
Manuel Moyano, Manuel I. Bermúdez, Antonia Ramírez \\ Universidad de Córdoba (España)
}

Disponible online 31 de diciembre de 2016

\begin{abstract}
Desde los atentados terroristas del 11S, el afrontamiento de la radicalización y el terrorismo yihadista por parte de las sociedades occidentales ha estado en primera línea de la agenda política interior y exterior. Durante este tiempo, se ha evidenciado la influencia que pueden desempeñar los dirigentes políticos en su interacción con las instituciones, la ciudadanía y las organizaciones terroristas. En la presente investigación se realiza un estudio de caso del discurso pronunciado por Barack Obama el 4 de junio de 2009 en la Universidad de El Cairo (“A New Beginning”). Para ello se examina su contenido y estructura interna utilizando como referente teórico-conceptual la clasificación de virtudes y fortalezas delimitada por Peterson y Seligman (2004) en el marco de la Psicología Positiva. Este discurso supuso un punto de inflexión en las relaciones entre Estados Unidos y el mundo árabo-musulmán, pudiendo considerarse un genuino ejercicio de comunicación positiva cuyas implicaciones están aún por determinar, dado que sigue ejerciendo influencia en la política interior y exterior de la administración Obama. Se concluye que, en un mundo globalizado y en red donde los riesgos y adversidades requieren abordajes innovadores, es necesaria más que nunca una comunicación social capaz de promover virtudes y fortalezas asociadas al optimismo, la esperanza, la confianza, la resistencia o la vitalidad.
\end{abstract}

Palabras clave: Psicología positiva, discurso, Obama, radicalización, terrorismo.

\begin{abstract}
Since the September 11 terrorist attacks, coping with radicalisation and jihadist terrorism has been at the forefront of the domestic and foreign policy agendas in Western societies. During this time, the influence that political leaders can exert in their interaction with institutions, citizens, and terrorist organizations has been demonstrated. We present a case study of the speech by Barak Obama on June 4, 2009 at the University of Cairo ("A New Beginning"). Its content and internal structure is analysed using the classification of virtues and strengths defined by Peterson and Seligman (2004) as a theoretical and conceptual reference within the framework of positive psychology. This speech marked a turning point in the relationship between the USA and the Arab-Muslim world and could be considered to be a genuine exercise in positive communication. Its implications are yet to be determined, because it continues to exert an influence on the Obama administration's domestic and foreign policy. In a globalized and networking world in which risks and adversities require innovative responses, more than ever we suggest that social communication capable of promoting virtues and strengths associated with optimism, hope, confidence, strength, or vitality is needed more than ever.
\end{abstract}

Key words: Positive Psychology, speech, Obama, radicalisation, terrorism.

Correspondencia: Manuel Moyano. Universidad de Córdoba. Facultad de Ciencias de la Educación. Avda. San Alberto Magno S/N. 14071 CÓRDOBA (España).E-mail: mmoyano@uco.es. E-mail del coautor Manuel Bermúdez: manuel.bermudez@uco.es; E-mail de la coatora Antonia Ramírez: ed1ragaa@uco.es 
Desde los atentados terroristas del 11S, pasando por la consiguiente intervención militar en Afganistán e Irak, los atentados terroristas del 11M en Madrid, 7J en Londres, y otros hitos históricos como la comunicación de la muerte de Bin Laden, la desafortunadamente denominada "Primavera Árabe" y el surgimiento del, también mal denominado Estado Islámico en Irak y Siria, han transcurrido tres lustros donde el afrontamiento de la radicalización y el terrorismo yihadista por parte de las sociedades occidentales han ocupado una prioridad en la agenda de interés público.

Todo lo expuesto anteriormente, junto con el escenario que se visualiza tras los recientes atentados yihadistas en suelo europeo y estadounidense, el éxodo de refugiados derivado de la guerra en Siria y los numerosos incidentes críticos de prejuicio y discriminación asociados a la polarización intergrupal en claves Islam-Occidente, corroboran la necesidad de prestar atención a la evolución de las percepciones entre el mundo árabo-musulmán y las sociedades occidentales (Kruglanski, Crenshaw, Post, y Victoroff, 2008; Moyano y Trujillo, 2013). Las características de esta amenaza han planteado nuevos retos a la seguridad de los Estados. Como es sabido, el terrorismo yihadista supone un entramado de organizaciones transnacionales en red, con una considerable legitimidad en determinados contextos geográficos y con un carácter deslocalizado acrecentado por las tecnologías de la información y la comunicación (Asal y Rethemeyer, 2008; Gambetta, 2005; Stern, 2004).

Durante este tiempo, se ha evidenciado de forma explícita la influencia que pueden desempeñar los dirigentes políticos en la interacción comunicativa, siempre dinámica, con las instituciones, la ciudadanía y las organizaciones terroristas (Bhatia, 2007; De Castella, McGarty y Musgrove, 2009; Kellner, 2007). En ese sentido, la comunicación social posterior a los atentados terroristas del $11 \mathrm{~S}$ estuvo muy vinculada a la percepción de la amenaza, y se realizó principalmente desde los supuestos básicos de la denominada doctrina Bush, caracterizada por los siguientes rasgos: (1) creencia en la legitimidad del Estado para determinar su política exterior e influir en el escenario internacional; (2) percepción de que existen grandes amenazas, (por ejemplo, el denominado Eje del Mal o rivales asimétricos de diferente tipo) que pueden ser afrontadas exclusivamente mediante nuevas y vigorosas políticas (por ejemplo, la guerra preventiva); (3) disposición a actuar unilateralmente cuando sea necesario; $y,(4)$ creencia de que, para conseguir la paz y la estabilidad, Estados Unidos debe hacer valer su primacía mundial (véase al respecto Jervis, 2003; Renshom y Suedfeld, 2007). Cuando Barack Obama ganó sus primeras elecciones presidenciales generó unas importantes expectativas tanto internamente como en la escena internacional. Sus habilidades retóricas fueron un componente central de su campaña y de su imagen, acaparando la atención de académicos y opinión pública (Coe y Reitzes, 2010). En relación al tópico que nos ocupa, existen varios discursos pronunciados durante sus mandatos que son especialmente significativos, tales como su primer discurso de investidura (2009), el discurso de la ceremonia de entrega del
Nobel de la Paz (2009), el discurso del anuncio de la muerte de Osama Bin Laden tras la Operación Gerónimo (2011), los discursos tras los atentados de Boston (2013) y Orlando (2016), el discurso sobre la estrategia antiterrorista (2015) o los relativos a las campañas de ataques contra el autodenominado Estado Islámico. Todos ellos, por citar sólo algunos, son fundamentales para comprender las líneas directrices de la administración Obama en el afrontamiento de la radicalización y el terrorismo, lo que algunos autores han caracterizado como la "Revolución de Obama" en lo que concierne al papel de Estados Unidos en el mundo y el restablecimiento de un nuevo orden internacional con el Medio Oriente (Indik, Liberthal y O'Hanlon, 2010). Sin embargo, el discurso pronunciado el 4 de junio de 2009 en la Universidad de El Cairo, "A New Beginning”, podría considerarse uno de los más relevantes en este ámbito. De hecho, podría decirse que esta alocución supuso, muy especialmente tras las política exterior derivada de los mandatos presidenciales de George W. Bush, un punto de inflexión en el manejo de las percepciones y las relaciones entre Estados Unidos y el mundo árabo-musulmán. El nuevo comienzo, para autores como Fawaz (2012) y Skidmore (2012) es indiscutible, evidenciado en la retirada de las tropas de Irak, la vuelta al diálogo con Irán y un orden internacional construido entre todos. Al mismo tiempo, el discurso, presentado paradójicamente en la capital del país del que procedían el mayor número de terroristas del $11 \mathrm{~S}$, tiene ciertos paralelismos con otras célebres disertaciones de presidentes estadounidenses en contextos a priori adversos, tales como el discurso de Kennedy en Berlín ("Ich bin ein Berliner", 1963), el discurso de Reagan en Moscú ("Moscow State University", 1988) o el más reciente discurso, también de Obama, en La Habana (2016).

Como en cualquier exposición oral, los componentes del lenguaje no verbal que acompañan y refuerzan las ideas transmitidas por Obama en "A New Beginning”, son un elemento a tener en cuenta. En este caso, el orador proyecta una imagen segura y un control absoluto sobre los aspectos que componen la comunicación no verbal, entendida ésta como los gestos, las posturas, las miradas, los tonos de voz y otras señales no verbales (Hall, Coats, y LeBeau, 2005; Hall, Schmid Mast y Latu, 2015). Entre otros aspectos cabe destacar su puesta en escena y su amplia sonrisa, que estimula que sea percibido como una persona sencilla y cercana. Cuando se dirige al público desde el atril ofrece una postura erguida y con las manos a ambos lados, sin mostrar tensión o nerviosismo. Al hablar, consigue un control permanente de las pausas, los silencios, el ritmo y el volumen. Además, de forma simultánea emplea gestos ilustradores y emblemáticos que refuerzan sus palabras y permiten apreciar muestras emotivas de respeto y agradecimiento. Pero sin duda, el aspecto más notable es el propio contenido del discurso, el cuál puede dividirse en siete bloques: (1) el extremismo violento; (2) el problema palestino; (3) el problema nuclear, con una referencia directa a Irán; (4) la democracia en el mundo islámico; (5) la libertad religiosa; (6) los derechos de las mujeres; y, (7) el desarrollo económico. Obama persigue 
un "nuevo comienzo" en las relaciones entre Estados Unidos y el mundo musulmán, sentar las bases de un lugar común de intereses y confianza e inhibir las percepciones negativas existentes. En todo la exposición trata de delinear asertivamente una separación categórica entre los radicales que se autoproclaman portavoces del Islam y la gran mayoría de musulmanes. Obama enfatiza con contundencia que Estados Unidos " $n o$ está, y nunca estará, en guerra con el Islam". La importancia de "A New Beginning" se ha analizado en diferentes trabajos desde una perspectiva pragma-dialéctica (Mohammed y Zarefsky, 2014), examinando los aspectos estratégicos de la oratoria de Obama (Kienpointner, 2013), vinculando las relaciones del discurso con la Psicología, la política exterior y la política interior de Obama (Soriano, 2014) o conceptualizando la doctrina derivada del mismo (Lewis, 2009).

En la presente investigación se realiza un estudio de caso de dicho discurso, considerando al mismo como un ejercicio de Psicología Positiva aplicada. Pero, ¿a qué nos referimos cuando hablamos de Psicología Positiva? Básicamente, la Psicología Positiva es un enfoque emergente que, en lugar de focalizarse en las limitaciones y en la fragilidad humana, prioriza el estudio de aquello que hace que la vida merezca ser vivida. Desde la Segunda Guerra Mundial, la Psicología se focalizó en lo negativo durante décadas, dedicando su potencial al abordaje de los problemas humanos y a sus posibles remedios (Gable y Haid, 2005; Seligman, 2003; Seligman, Rashid, y Parks, 2006). Sin embargo, las restricciones de este sesgo han ido poniéndose de manifiesto en los últimos años. Así, en el año 2000, la revista American Psychologist publicó un monográfico sobre Psicología Positiva, movimiento liderado principalmente por Seligman y Csikzentmihalyi, cuyo interés se centra no sólo en la eliminación del malestar o la superación de los déficits, sino en la promoción del funcionamiento óptimo y el fomento del bienestar (Seligman y Csikszentmihalyi, 2000). Aunque esta perspectiva no ha estado exenta de críticas (Coyne y Tennen, 2010; Lazarus, 2003; Miller, 2008; Pérez-Álvarez, 2012), también se han puesto en valor las evidencias empíricas disponibles al respecto y la sólida agenda de investigación sobre las emociones

\section{Figura 1}

Virtudes y fortalezas humanas (adaptado de Peterson y Seligman, 2004).

\begin{tabular}{|c|c|}
\hline Virtudes & Fortalezas \\
\hline Sabiduría: fortalezas cognitivas para adquirir y emplear el conocimiento. & $\begin{array}{l}\text { - Creatividad: pensar nuevas formas de hacer las cosas y conceptos. } \\
\text { - Curiosidad: interesarse por las cosas, explorar, indagar. } \\
\text { - Perspectiva: comprender el mundo, ofrecer sabios consejos. } \\
\text { - Juicio: calibrar y tener en cuenta toda la evidencia disponible. } \\
\text { - Deseo de aprender: interés en aprender de forma sistemática. }\end{array}$ \\
\hline $\begin{array}{l}\text { Valor: fortalezas emocionales, voluntad para lograr objetivos ante situaciones adversas e } \\
\text { incertidumbre. }\end{array}$ & $\begin{array}{l}\text { - Perseverancia: completar las tareas que se empiezan. } \\
\text { - Valentía: no amedrentarse por la amenaza o la adversidad. } \\
\text { - Honestidad: comportarse de forma genuina e íntegra. } \\
\text { - Vitalidad: vivir de forma apasionada y con energía. }\end{array}$ \\
\hline Humanidad: fortalezas interpersonales, acercamiento a los otros, cultivar las relaciones. & $\begin{array}{l}\text { - Inteligencia social: comprender el mundo social. } \\
\text { - Bondad: ayudar y cuidar de los demás. } \\
\text { - Amor: valorar las relaciones cercanas. } \\
\end{array}$ \\
\hline Justicia: fortalezas cívicas subyacentes a una vida comunitaria saludable. & $\begin{array}{l}\text { - Liderazgo: organizar la actividad de grupo. } \\
\text { - Equidad: tratar de forma equitativa y justa. } \\
\text { - Trabajo en equipo: ser un buen miembro del equipo. }\end{array}$ \\
\hline $\begin{array}{l}\text { Templanza: fortalezas protectoras de los excesos y facilitan expresión y moderación de } \\
\text { apetitos y necesidades. }\end{array}$ & $\begin{array}{l}\text { - Perdón: perdonar a otros. } \\
\text { - Autorregulación: regular sentimientos y acciones. } \\
\text { - Prudencia: elegir las acciones con cuidado. } \\
\text { - Modestia: no sobrevalorarse a uno mismo. }\end{array}$ \\
\hline Trascendencia: fortalezas que proporcionan significado y unión con el universo. & $\begin{array}{l}\text { - Espiritualidad: creencias sobre el propósito y el sentido vital. } \\
\text { - Aprecio de la belleza: ser consciente de la excelencia. } \\
\text { - Esperanza: expectativas de un futuro positivo. } \\
\text { - Gratitud: agradecimiento por las cosas buenas. } \\
\text { - Humor: apreciar el lado positivo de la vida, reir. }\end{array}$ \\
\hline
\end{tabular}

y cogniciones positivas, así como su relación con la salud y el bienestar (Vázquez, 2013; Vázquez y Hervás, 2008). Siguiendo a Peterson (2006), tres tópicos componen el centro de interés de la Psicología Positiva: (1) las experiencias subjetivas positivas (p.ej. el placer o la felicidad); (2) los rasgos individuales positivos (p.ej. fortalezas o talentos); y, (3) las instituciones (p.ej. familias, escuelas). Asimismo, Meyers, Van Woerkom y Bakker (2012) realizaron una revisión sistemática de las investigaciones sobre los efectos de las intervenciones positiva aplicadas al contexto organizacional. Además de encontrar que las intervenciones de la psicología positiva parecen ser una herramienta prometedora para mejorar el rendimiento y el bienestar de los miembros de la organización, definieron dichas intervenciones psicológicas positivas como (1) aquellas actividades intencionales o métodos basados en el cultivo de experiencias subjetivas positivas, (2) el desarrollo de rasgos individuales positivos, y (3) la promoción de instituciones positivas basadas en el civismo y la virtud.

Partiendo de estos referentes teóricos cabría preguntarse lo siguiente ¿hasta qué punto los líderes políticos, a través de su comunicación social, pueden promover esas experiencias subjetivas en la sociedad? ¿Puede dicha comunicación social convertirse en un catalizador para desarrollar e inspirar rasgos individuales positivos? ¿Pueden promoverse, de esta forma, instituciones y modelos sociales basados en la virtud? ¿Pueden liderar la construcción de sociedades resistentes, maduras y capaces de resolver conflictos en situaciones adversas? El objetivo general de esta investigación es analizar un discurso político de trascendencia internacional ("A New Beginning") utilizando como referencia el modelo teórico-conceptual de virtudes y fortalezas propuesto por Peterson y Seligman (2004) en el marco de la Psicología Positiva. Estos autores propusieron 24 fortalezas humanas clasificadas en seis subgrupos de virtudes a partir de una revisión bibliográfica exhaustiva (ver figura 1). Aunque Peterson y Seligman (2004) precisaron que esta taxonomía era una clasificación preliminar abierta y flexible, desde entonces y hasta ahora han ido diseñando instrumentos de medida que permiten recoger datos en poblaciones muy 
diversas y conocer, no sólo si existen o no tales virtudes y fortalezas, sino los diferentes grados que pueden presentar. En última instancia, mediante la presente investigación se tratará de visibilizar cómo el mensaje comunicativo analizado integra elementos con un carácter positivo, utilizando como indicadores dichas virtudes y fortalezas.

\section{Método}

En este apartado se concretan los aspectos básicos de la metodología llevada a cabo en la investigación: diseño metodológico, material utilizado, categorías de análisis y procedimiento seguido.

\section{Diseño}

Se utilizó una metodología cualitativa basada en el análisis de contenido. Éste se ubica, según López (2002, p. 174), “en el ámbito de la investigación descriptiva" y pretende "descubrir los componentes básicos de un fenómeno determinado extrayéndolos de un contenido dado a través de un proceso que se caracteriza por el intento de rigor en la medición". En esta misma dirección, el análisis de contenido es una técnica de investigación cuya forma es replicable y de dónde se obtienen inferencias válidas de un texto o de otras fuentes (Krippendorf, 2013).

\section{Material}

La información fue obtenida mediante la consulta del contenido audiovisual del discurso “A New Beginning”, así como de su correspondiente transcripción y traducción (puede consultarse en www.whitehouse.gov).

\section{Categorías de análisis}

A fin de extraer las diferentes categorías de análisis, se examinó y clasificó el contenido de esta fuente secundaria utilizando como referente teórico-conceptual las seis virtudes y fortalezas asociadas propuestas por Peterson y Seligman (2004). Se empleó el software ATLAS.ti v.6 como recurso técnico.

\section{Procedimiento}

Para consensuar la identificación, selección y discriminación de los pasajes del discurso que podían vincularse con las seis virtudes, se realizó un proceso de consulta externa mediante técnica Delphi a tres expertos en Psicología Positiva. En dicho proceso se les pidió que localizaran indicadores de las virtudes y fortalezas del modelo en los diferentes fragmentos del texto. Algunos pasajes del discurso podían vincularse a diferentes categorías. Con el fin de solventar las discrepancias que surgieron en la consulta, al final del proceso se realizó un grupo focal coordinado por los investigadores, y en el que los tres expertos llegaron a una convergencia de criterio y a la consiguiente versión final. Se mantuvieron exclusivamente aquellos indicadores de virtudes y fortalezas en los que tras el grupo focal hubo consenso entre los tres expertos.

\section{Resultados}

El marcado carácter positivo del discurso se puede visualizar en la figura 2, en la que se recopilan los pasajes del texto en los que hubo convergencia inter-observadores y que pueden

Tabla 2

Categorías de virtudes y fortalezas (Peterson y Seligman, 2004) e indicadores asociados en "A New Beginning".

\begin{tabular}{|c|c|}
\hline Virtudes y fortalezas & Indicadores \\
\hline $\begin{array}{l}\text { Sabiduría } \\
\text { - creatividad } \\
\text { - curiosidad } \\
\text { - perspectiva } \\
\text { - juicio } \\
\text { - deseo aprender }\end{array}$ & $\begin{array}{l}\text { "La experiencia guía mi convicción de que esa alianza entre Estados Unidos y el Islam se debe basar en lo que es el Islam, no en lo que no es". / "El Sagrado Corán } \\
\text { enseña que quien mata a un inocente, mata a toda la humanidad; y quien salva a una persona, salva a toda la humanidad. La religión perdurable de más de mil millones de } \\
\text { personas es mucho más fuerte que el odio intransigente de unos pocos. El Islam no es parte del problema en la lucha contra el extremismo violento, es una parte importante } \\
\text { en el avance hacia la paz". I "Si vemos este conflicto solamente de un lado o del otro, entonces no podemos ver la verdad: la única solución es que las aspiraciones de } \\
\text { ambos lados las satisfagan dos estados, donde los israelies y los palestinos tengan paz y seguridad". / "Es más fácil comenzar guerras que llevarlas a su fin. Es más fácil } \\
\text { culpar a otros que mirar hacia adentro, ver las diferencias en los demás que las semejanzas. Pero debemos escoger el camino correcto, no el camino fácill". / "Hay una } \\
\text { regla central en toda religión: tratar a los demás como uno quisiera ser tratado". }\end{array}$ \\
\hline $\begin{array}{l}\text { Valor } \\
\text { - perseverancia } \\
\text { - valentía } \\
\text { - honestidad } \\
\text { - vitalidad }\end{array}$ & $\begin{array}{l}\text { "Estoy convencido de que para progresar, debemos decir abiertamente lo que pensamos, y demasiadas veces, eso se dice solamente detrás de puertas cerradas". I } \\
\text { "Haremos frente sin descanso a los extremistas violentos que representan una grave amenaza para nuestra seguridad, porque rechazamos lo mismo que rechaza la gente } \\
\text { de todos los credos: el asesinato de hombres, mujeres y niños inocentes. Y es mi deber principal como Presidente proteger al pueblo estadounidense". / "Asi como Estados } \\
\text { Unidos no puede tolerar la violencia a manos de extremistas, nunca debemos cambiar nuestros principios". I "Tenemos el poder de crear el mundo que queremos, pero } \\
\text { sólo si tenemos la valentia de crear un nuevo comienzo". }\end{array}$ \\
\hline $\begin{array}{l}\text { Humanidad } \\
\text { - inteligencia social } \\
\text { - bondad } \\
- \text { amor }\end{array}$ & $\begin{array}{l}\text { "Es parte de mi responsabilidad como Presidente de Estados Unidos luchar contra los estereotipos negativos del Islam dondequiera que surjan. Pero ese mismo principio } \\
\text { debe aplicarse a la percepción musulmana de Estados Unidos". " "En Ankara dejé en claro que Estados Unidos no está y nunca estará en guerra contra el Islam". " "Es } \\
\text { una historia con una verdad muy simple: la violencia es un callejón sin salida". " "Nuestras hijas pueden contribuir tanto a la sociedad como nuestros hijos, y nuestra } \\
\text { prosperidad común se puede promover si permitimos a toda la humanidad - hombres y mujeres - a lograr todo su potencial". " "Todos nosotros compartimos este mundo } \\
\text { sólo por un breve periodo. El asunto es si vamos a pasar este tiempo centrados en lo que nos separa o si nos comprometeremos a realizar un esfuerzo -un esfuerzo } \\
\text { sostenido- con el fin de encontrar terreno común, de concentrarnos en el futuro que queremos para nuestros hijos y de respetar la dignidad de todos los seres humanos". }\end{array}$ \\
\hline $\begin{array}{l}\text { Justicia } \\
\text { - liderazgo } \\
\text { - equidad } \\
\text { - trabajo en equipo }\end{array}$ & 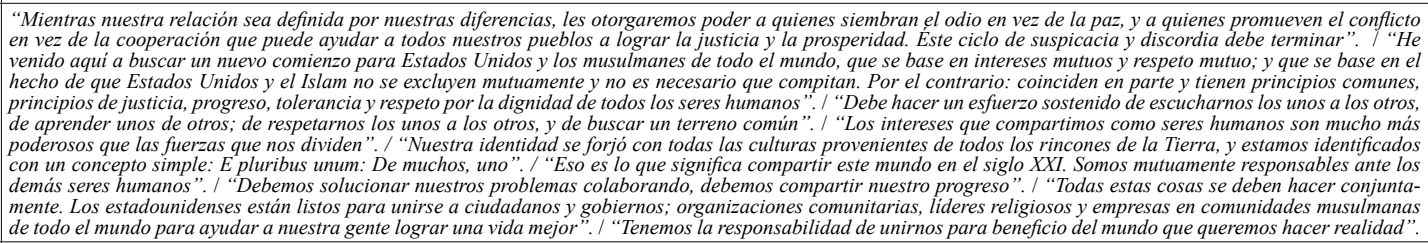 \\
\hline $\begin{array}{l}\text { Templanza } \\
\text { - perdón } \\
\text { - autorregulación } \\
\text { - prudencia } \\
\text { - modestia }\end{array}$ & $\begin{array}{l}\text { "También sabemos que el poderio militar por sí solo no va a resolver los problemas en Afganistán y Pakistán". I "Creo que los acontecimientos en Irak han recordado a } \\
\text { Estados Unidos que es necesario usar la diplomacia y promover consenso a nivel internacional para resolver nuestros problemas cuando sea posible". / "El ll de Sep- } \\
\text { tiembre fue un trauma enorme para nuestro pais. El temor y la ira que causó son comprensibles, pero en algunos casos, nos llevó a actuar en contra de nuestros ideales. } \\
\text { Estamos tomando medidas concretas para cambiar de curso. He prohibido inequivocamente el uso de tortura por Estados Unidos y he ordenado que se clausure la prisión } \\
\text { en la bahía de Guantánamo para comienzos del próximo año". " "No es señal de valentia ni fuerza lanzar cohetes contra niños que duermen, ni hacer estallar ancianas } \\
\text { en un autobús. Así no se obtiene autoridad moral; así se renuncia a ella". I "Entre algunos musulmanes, hay una tendencia preocupante de medir las creencias propias } \\
\text { en base al rechazo de las de los demás". " "Hay quienes están ansiosos por avivar las llamas de la división e impedir el progreso. Hay quienes sugieren que no vale la } \\
\text { pena; alegan que estamos destinados a discrepar y las civilizaciones están condenadas a tener conflictos. El escepticismo embarga a muchos más. Hay tanto temor, tanta } \\
\text { desconfianza. Pero si optamos por ser prisioneros del pasado, entonces nunca avanzaremos". }\end{array}$ \\
\hline $\begin{array}{l}\text { Trascendencia } \\
\text { - espiritualidad } \\
\text { - aprecio belleza } \\
\text { - esperanza } \\
\text { - gratitud } \\
\text { - humor }\end{array}$ & 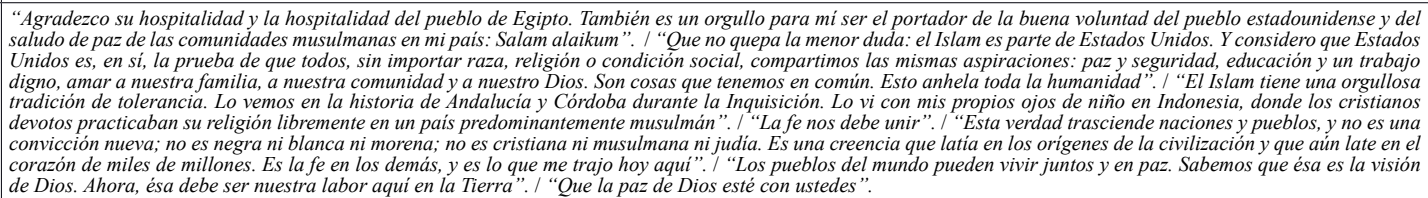 \\
\hline
\end{tabular}


interpretarse como indicadores de las virtudes y fortalezas del modelo de Peterson y Seligman (2004).

\section{Discusión y conclusiones}

En la presente investigación se ha realizado un análisis cualitativo de "A New Beginning", considerándolo como un ejemplo paradigmático de Psicología Positiva aplicado al afrontamiento de situaciones adversas, en este caso, a la resolución de conflictos. Como hemos expuesto, a lo largo del texto aparecen numerosos pasajes que pueden concebirse como indicadores de las virtudes y fortalezas formuladas por Peterson y Seligman (2004) desde el marco de la Psicología Positiva. Este discurso presidencial, al igual que muchos otros, se configura como un instrumento que permite no sólo transmitir una información, sino también ofrecer la visión del mundo que tiene el dirigente político en cuestión. En el caso que nos ocupa, el lenguaje empleado por el presidente Obama, construye una realidad, una realidad "verdadera", que es ofrecida al mundo como la nueva imagen de Estados Unidos en las relaciones internacionales, al objeto de cambiar la percepción sobre el país más poderoso del mundo en las últimas décadas y de buscar un nuevo escenario, posiblemente con nuevos roles.

Las implicaciones del discurso de Obama en el Cairo, a todas luces un habilidoso ejercicio de comunicación social, están aún por determinar, dado que sigue ejerciendo influencia y está cargado de sentido en la actualidad de las relaciones internacionales. En base a esta ambiciosa declaración de intenciones, podría realizarse una propuesta preliminar de la doctrina Obama en el afrontamiento de la radicalización y el terrorismo yihadista. Previamente definiremos el concepto de doctrina como un sistema integrado de opiniones y creencias que persiguen influir en las actitudes, emociones y comportamientos de los miembros de un grupo social, con una estructura discursiva y una amplitud de miras sobre la interpretación de la condición humana y el mundo y, expresado, habitualmente, en discursos o comunicaciones de carácter político (Jervis, 2003; Renshom y Suedfeld, 2007). Básicamente, la doctrina Obama en el ámbito del antiterrorismo tendría los siguientes rasgos: (1) preventiva, más que reactiva; (2) multilateral, más que unilateral; (3) negociadora, tendente a cohesionar al endogrupo y al exogrupo en una identidad social común, tendente a inhibir la polarización; (4) promotora de la democracia de forma persuasiva, pero enfatizando la diplomacia; (5) énfasis en la clarificación asertiva del enemigo potencial, en el aislamiento de los radicales y en visibilizar las diferencias entre terrorismo e Islam; (6) énfasis en favorecer los recursos psicosociales de afrontamiento de personas y comunidades, especialmente en entornos adversos; y, por último, (7) propósito de promover la "reducción del prejuicio" entre los colectivos en conflicto, en detrimento de la promoción de la metáfora de "guerra contra el terror".

Esta caracterización es sólo una propuesta inicial y metodológicamente restringida, pero que podría ser esgrimida para establecer un esquema de la doctrina Obama en contraposición con el eje vertebrador de la comunicación y la política sobre antiterrorismo en los mandatos de George W. Bush. También podría ponerse en contraposición y antagonismo con la comunicación social y las expectativas generadas al respecto por el presidenciable Donald Trump de cara a las elecciones de 2016.

La pregunta es: ¿puede esta perspectiva positiva, que caracteriza a la docrina Obama, tener alguna aplicación en el afrontamiento del terrorismo? ¿Puede la promoción y difusión de estos aspectos positivos tener efectos beneficiosos en la prevención y el antiterrorismo? ¿Puede suponer perjuicios? Aunque a día de hoy aún disponemos de una escasez de evidencias empíricas sobre la efectividad de las medidas antiterroristas (veáse una revisión sistemática y una colaboración Campbell al respecto en Lum, Kennedy, y Sherley, 2008), podríamos aventurar que, tanto la doctrina Bush como la doctrina Obama aquí caracterizada, tendrían fortalezas y debilidades, y serían deudoras de su tiempo y circunstancias. Las políticas centradas en la comunicación social de la amenaza, ejemplificadas manifiestamente por la doctrina Bush, pueden suponer un efecto positivo a corto plazo en pos de determinados objetivos. No obstante, a largo plazo también pueden generar efectos negativos tales como el deterioro de la imagen pública (en este caso de Estados Unidos), la vulneración de los fundamentos del Estado de Derecho, inflexibilidad para afrontar una amenaza tan difusa e innovadora como el terrorismo yihadista o la expansión de un alarmismo excesivo que, paradójicamente, puede robustecer a los terroristas como "enemigos", facilitando el amedrentamiento que persiguen estimular. Además, a día de hoy existen evidencias empíricas para afirmar que, aunque desde un punto de vista psicosocial las relaciones funcionales no son simples, la percepción de amenaza (evaluada mediante indicadores de conflicto percibido, expectativas de mortalidad, humillación, privación relativa, prejuicio o discriminación) favorece la legitimación de la violencia del endogrupo hacia exogrupos potenciales y puede ser contraproducente en términos de polarización intergrupal. Algo que, en definitiva, puede potenciar la radicalización, y en última instancia, la captación y el reclutamiento terrorista a la largo plazo (Kruglanski et al., 2014; Moyano y Trujillo, 2013; Pyszczynski et al., 2006; Victoroff, 2005).

Por otra parte, podríamos decir que la doctrina Obama, ejemplificada en "A New Beginning" y con un enfoque eminentemente positivo, estaría caracterizada por la petición de responsabilidades mutuas, la reducción del prejuicio intergrupal y el uso de la fuerza exclusivamente cuando sea necesario. La misma promueve los aspectos positivos del desarrollo humano, las relaciones intergrupales conciliadoras basadas en la confianza y el respeto, así como la búsqueda de la justicia, la igualdad y el bienestar para todos los seres humanos. Sin embargo, en cierto sentido, este posicionamiento también podría ser interpretado, por ciertos sectores y en determinados escenarios, como una escandalosa muestra de debilidad y 
"buenismo". Además, las elevadas expectativas sobre unos y otros podrían generar frustración, desasosiego e incertidumbre en caso de que el ambicioso horizonte que se anhela no se convierta en realidad.

Podríamos concluir que, en términos de antiterrorismo, sería conveniente complementar un modelo hermético y sesgado de "guerra contra el terror" con otros objetivos integradores centrados en aspectos eminentemente positivos (Kruglanski, Crenshaw, Post, y Victoroff, 2008; Moyano y Trujillo, 2013). El abordaje de la radicalización y el terrorismo yihadista debe incluir tales aspectos, no ya sólo por la legitimidad moral que intrínsecamente conllevan, sino porque a largo plazo pueden resultar en estrategias y estilos de liderazgo mucho más eficaces y efectivos que un abordaje reactivo y reduccionista centrado únicamente en la "metáfora de la guerra" ejemplificada por la doctrina Bush. Así, es previsible que una comunicación social positiva centrada en la reducción del prejuicio, la prevención de la radicalización de los actores, la proactividad y la anticipación, sea más efectiva para controlar las reacciones violentas del exogrupo, vertebrar una sólida red de alianzas, modular estados de opinión polarizados, dividir a potenciales enemigos, $\mathrm{y}$ en definitiva, evitar ciclos de odio anclados en la humillaciónvenganza que poco van servir para mejorar la seguridad global a largo plazo. De esta manera, estaremos realmente en el camino de construir sociedades más fuertes y resistentes ante la adversidad que representan la radicalización y el terrorismo.

Aproximarnos analíticamente a las políticas antiterroristas exclusivamente a través del examen del contenido y las características de los discursos políticos es un posicionamiento reduccionista. Sin embargo, como hemos visto, la retórica de los dirigentes puede modular la percepción de amenaza, la cohesión intergrupal, la construcción del enemigo y la legitimación de determinadas políticas más o menos beligerantes y reactivas. En un mundo globalizado y en red donde los riesgos y adversidades requieren abordajes innovadores es necesaria más que nunca una comunicación social capaz de promover virtudes y fortalezas asociadas al optimismo, la esperanza, la confianza, la resistencia o la vitalidad. Y por supuesto, velar porque las palabras siempre vengan acompañadas de hechos. Es ineludible, por tanto, seguir prestando atención a estos procesos de influencia social.

\section{Referencias}

1. Asal, V. y Rethemeyer, R.K. (2008). The nature of the beast: Terrorist organizational characteristics and organizational lethality. Journal of Politics, 70, 437-49. https://doi. org/10.1017/S0022381608080419

2. Bhatia, A. (2007). Religious metaphor in the discourse of illusion: George W. Bush and Osama bin Laden. World Englishes, 26, 507-524. https://doi.org/10.1111/j.1467971X.2007.00525.x

3. Coe, K. y Reitzes, M. (2010). Obama on the Stump: Features and Determinants of a Rhetorical Approach.
Presidential Studies Quarterly, 40, 391-413. https://doi. org/10.1111/j.1741-5705.2010.03777.x

4. Coyne, J.C., y Tennen, H. (2010). Positive psychology in cancer care: Bad science, exaggerated claims, and unproven medicine. Annals of Behavioral Medicine, 39, 16-26. https://doi.org/10.1007/s12160-009-9154-z

5. De Castella, K., McGarty, C. y Musgrove, L. (2009). Fear Appeals in Political Rhetoric about Terrorism: An Analysis of Speeches by Australian Prime Minister Howard. Political Psychology, 30, 1-26. https://doi.org/10.1111/j.14679221.2008.00678.x

6. Fawaz, G. (2012). The Obama Approach to the Middle East: The end of the American Moment? The Royal Institute of International Affairs, 89 (2). Recuperado de https://www. chathamhouse.org/publications/ia/archive/view/189843

7. Gable, S. L. y Haid, J. (2005). What (and Why) Is Positive Psychology? Review of General Psychology, 9, 103-110. https://doi.org/10.1037/1089-2680.9.2.103

8. Gambetta, G. (2005). Making Sense of Suicide Missions. New York: Oxford University Press. https://doi. org/10.1093/acprof:oso/9780199276998.001.0001

9. Hall, J.A., Coats, E.J., y LeBeau, L. S. (2005). Nonverbal behavior and the vertical dimensions of social relations: A meta-analysis. Psychological Bulletin, 131, 898-924. https://doi.org/10.1037/0033-2909.131.6.898

10. Hall, J.A., Schmid Mast, M. y Latu, I. (2015). The vertical dimension of social relations and accurate interpersonal perception: A meta-analysis. Journal of Nonverbal Behavior, 39, 131-163. https://doi.org/10.1007/s10919014-0205-1

11. Indik, M., Liberthal, K. y O'Hanlon, M. (2010). Bending History. Barack Obama's Foreign Policy. Maryland: Brooking Institution Press.

12. Jervis, R. (2003). Understanding the Bush Doctrine. Political Science Quarterly, 118, 365-388. https://doi. org/10.1002/j.1538-165X.2003.tb00398.x

13. Kellner, D. (2007). Bushspeak and the Politics of Lying: Presidential Rhetoric in the "War on Terror". Presidential Studies Quarterly, 37, 622-645. https://doi.org/10.1111/ j.1741-5705.2007.02617.x

14. Kienpointner, M. (2013). Strategic maneuvering in the political rhetoric of Barack Obama. Journal of Language and Politics, 12, 357-377. https://doi.org/10.1075/ jlp.12.3.03kie

15. Krippendorf, K. (2013). Content Analysis: An Introduction to its methodology. Londres: SAGE.

16. Kruglanski, A.W., Crenshaw, M., Post, J.M., y Victoroff, J. (2008). What Should This Fight Be Called? Metaphors of Counterterrorism and Their Implications. Psychological Science in the Public Interest, 8, 97-133. https://doi. org/10.1111/j.1539-6053.2008.00035.x

17. Kruglanski, A. W., Gelfand, M., Bélanger, J. J., Sheveland, A., Hettiarachchi, M., y Gunaratna, R. (2014). The 
Psychology of Radicalization and Deradicalization: How Significance Quest Impacts Violent Extremism. Advances in Political Psychology, 35, 9-93. https://doi.org/10.1111/ pops. 12163

18. Lazarus, R. S. (2003). Does the positive psychology movement have legs? Psychological Inquiry, 14, 93-109. https:// doi.org/10.1207/S15327965PLI1402_02

19. Lewis, P.M. (2009). La doctrina Obama: un análisis del discurso de Obama en El Cairo. Estudios Internacionales, 163, 129-133.

20. López, F. (2002). El análisis de contenido como método de investigación. XXI, Revista de Educación, 4, 169-179.

21. Lum, C., Kennedy, L. y Sherley, A. (2008). Is counter-terrorism policy evidence-based? What works, what harms, and what is unknown. Psicothema, 20, 35-42.

22. Meyers, M.C., Van Woerkom, M., y Bakker, A.B. (2013). The added value of the positive: A literature review of positive psychology interventions in organizations. European Journal of Work and Organizational Psychology, 22, 618632. https://doi.org/10.1080/1359432X.2012.694689

23. Miller, A. (2008), A Critique of Positive Psychology - or 'The New Science of Happiness'. Journal of Philosophy of Education, 42, 591-608. https://doi.org/10.1111/j.14679752.2008.00646.x

24. Mohammed, D., y Zarefsky, D. (2014). Pragma-Dialectical Analysis of Rhetorical Texts: The Case of Barack Obama in Cairo. Rhetorical Perspectives on Argumentation, 24, 253265. https://doi.org/10.1007/978-3-319-05485-8 20

25. Moyano, M., y Trujillo, H.M. (2013). Radicalización islamista y terrorismo. Claves psicosociales. Granada: Editorial de la Universidad de Granada, EUG.

26. Pérez-Álvarez, M. (2012). La Psicología Positiva: una moda simpática. Papeles del Psicólogo, 33, 183-201.

27. Peterson, C., y Seligman, M.E.P. (2004). Character strengths and virtues: A handbook and classification. Washington, DC: American Psychological Association.

28. Peterson, C. (2006). A primer in positive psychology. New York: Oxford University Press.

29. Pyszczynski, T., Abdollahi, A., Solomon, S., Greenberg, J., Cohen, F., y Weise, D. (2006). Mortality salience, martyrdom, and military might: The Great Satan versus the axis of evil. Personality and Social Psychology Bulletin, 32, 525537. https://doi.org/10.1177/0146167205282157
30. Renshon, S.A., y Suedfield, P. (Eds.) (2007). Understanding the Bush Doctrine: Psychology and Strategy in an Age of Terrorism. New York-London: Routledge. Taylor \& Francis Group.

31. Seligman, M.E.P. (2003). La auténtica felicidad. Barcelona, Ediciones Vergara.

32. Seligman, M.E.P., y Csikszentmihalyi, M. (2000). Positive psychology: An introduction. American Psychologist, 55, 5-14.

33. Seligman, M.E.P., Rashid, T. y Parks, A.C. (2006). Positive Psychotherapy. American Psychologist, 61, 774-788. https://doi.org/10.1037/0003-066X.61.8.774

34. Skidmore, D. (2012). The Obama Presidency and US Foreign Policy: Where is the Multilateralism? International Studies Perspective, 13, 43-64. https://doi.org/10.1111/ j.1528-3585.2011.00454.x

35. Soriano, M.L. (2014). Tres frentes del discurso de Barack Obama en el ámbito del derecho y la política: los aliados, los musulmanes y los inmigrantes. Revista Internacional de Pensamiento Político, 9, 105-124.

36. Stern, J. (2004). Terror in the name of God. New York, NY: Ecco.

37. Vázquez, C. (2013). La psicología positiva y sus enemigos: una réplica en base a la evidencia científica. Papeles del Psicólogo, 34, 91-115.

38. Vázquez, C. y Hervás, G. (Eds.) (2008). Psicología positiva aplicada. Bilbao: DDB.

39. Victoroff, J. (2005). The mind of the terrorist. A review and critique of psychological approaches. Journal of Conflict Resolution, 49, 3-42. https://doi. org/10.1177/0022002704272040

RECIBIDO: $10 / 08 / 2016$ REVISADO: $18 / 09 / 2016$ ACEPTADO: 20/10/2016 